\title{
Systems biological assessment of immunity to mild versus severe COVID-19 infection in humans
}

\author{
Prabhu S. Arunachalam ${ }^{1 *}$, Florian Wimmers ${ }^{1 *}$, Chris Ka Pun Mok $^{2 *}$, Ranawaka A. P. M. Perera ${ }^{3 *}$, Madeleine \\ Scott $^{1,4} \uparrow$, Thomas Hagan' ${ }^{1}$, Natalia Sigal ${ }^{1}+$, Yupeng Feng ${ }^{1} \uparrow$, Laurel Bristow ${ }^{5}$, Owen Tak-Yin Tsang ${ }^{6}$, Dhananjay \\ Wagh $^{7}$, John Coller ${ }^{7}$, Kathryn L. Pellegrini ${ }^{8}$, Dmitri Kazmin', Ghina Alaaeddine ${ }^{5}$, Wai Shing Leung ${ }^{6}$, Jacky Man \\ Chun Chan $^{6}$, Thomas Shiu Hong Chik ${ }^{6}$, Chris Yau Chung Choi ${ }^{6}$, Christopher Huerta ${ }^{5}$, Michele Paine \\ McCullough $^{5}$, Huibin Lv ${ }^{2}$, Evan Anderson ${ }^{9}$, Srilatha Edupuganti ${ }^{5}$, Amit A. Upadhyay ${ }^{8}$, Steve E. Bosinger ${ }^{8,10}$, \\ Holden Terry Maecker', Purvesh Khatri ${ }^{1,4}$, Nadine Rouphael ${ }^{5}$, Malik Peiris ${ }^{2,3}$, Bali Pulendran ${ }^{1,11,12}$ *
}

${ }^{1}$ Institute for Immunity, Transplantation and Infection, School of Medicine, Stanford University, Stanford, CA 94305, USA. ${ }^{2}$ HKU-Pasteur Research Pole, School of Public Health, HKU Li Ka Shing Faculty of Medicine, The University of Hong Kong (HKU), Hong Kong. ${ }^{3}$ Centre of Influenza Research, School of Public Health, HKU Li Ka Shing Faculty of Medicine, The University of Hong Kong, Hong Kong. ${ }^{4}$ Center for Biomedical informatics, Department of Medicine, Stanford University School of Medicine, Stanford, CA 94305, USA. ${ }^{\mathrm{H}}$ ope Clinic of the Emory Vaccine Center, Department of Medicine, Division of Infectious Diseases, Emory University School of Medicine, Decatur, GA 30030, USA. ${ }^{6}$ nfectious Diseases Centre, Princess Margaret Hospital, Hospital Authority of Hong Kong, Hong Kong. ${ }^{7}$ Stanford Functional Genomics Facility, Stanford University School of Medicine, Stanford, CA 94305, USA. ${ }^{8}$ Emory Vaccine Center, Yerkes National Primate Research Center, Atlanta, GA 30329, USA. ${ }^{9}$ Department of Pediatrics, Division of Infectious Disease, Emory University School of Medicine, Atlanta, GA 30322, USA. ${ }^{10}$ Department of Pathology and Laboratory Medicine, Emory University, Atlanta, GA 30329, USA. ${ }^{11}$ Department of Pathology, Stanford University School of Medicine, Stanford, CA 94305, USA. ${ }^{12}$ Department of Microbiology and Immunology, Stanford University School of Medicine, Stanford, CA 94305, USA.

*These authors contributed equally to this work. †These authors contributed equally to this work.

‡Corresponding author. Email: bpulend@stanford.edu

COVID-19 represents a global crisis, yet major knowledge gaps remain about human immunity to SARSCoV-2. We analyzed immune responses in 76 COVID-19 patients and 69 healthy individuals from Hong Kong and Atlanta. In PBMCs of COVID-19 patients, there was reduced expression of HLA-DR and proinflammatory cytokines by myeloid cells, and impaired mTOR-signaling and IFN- $\alpha$ production by plasmacytoid DCs. In contrast, there were enhanced plasma levels of inflammatory mediators, including EN-RAGE, TNFSF14, and oncostatin-M, which correlated with disease severity and increased bacterial products in human plasma. Single-cell transcriptomics revealed no type-I IFN, reduced HLA-DR in myeloid cells of severe patients, and transient expression of IFN-stimulated genes. This was consistent with bulk PBMC transcriptomics, and transient, low plasma IFN- $\alpha$ levels during infection. These results reveal mechanisms and potential therapeutic targets for COVID-19.

The recent emergence of the SARS-coronavirus 2 (SARS-CoV2) in Wuhan, China, in December 2019, and its rapid international spread poses a global pandemic. Research has moved rapidly in isolating, sequencing and cloning the virus, developing diagnostic kits, and testing candidate vaccines. However, major questions remain about the dynamic interaction between the human immune system and the SARS-CoV-2 virus.

COVID-19 presents with a spectrum of clinical phenotypes, with most patients exhibiting mild-to-moderate symptoms, and $15 \%$ progressing typically within a week to severe or critical disease that requires hospitalization (1), and a minority of those develop acute respiratory disease syndrome (ARDS) requiring mechanical ventilation. Epidemiological data so far suggest that COVID-19 has a case fatality rate several times greater than that of seasonal influenza (1). The elderly and individuals with underlying medical comorbidities such as cardiovascular disease, diabetes mellitus, chronic lung disease, chronic kidney disease, obesity, hypertension or cancer have a much higher mortality rate than healthy young adults (2). The underlying causes are unknown, but may be due to an impaired interferon response, and dysregulated inflammatory responses as observed with other zoonotic coronavirus infections such as Severe Acute Respiratory Syndrome (SARS) and Middle East Respiratory Syndrome (MERS) (3). Current research is uncovering how the adaptive immune response to SARS-CoV-2 is induced with optimal functional capacities to clear SARS-CoV viral infection (4-6).

Understanding the immunological mechanisms underlying the diverse clinical presentations of COVID-19 is a critical step in the design of rational therapeutic strategies. Recent reports suggest that COVID-19 patients are characterized by lymphopenia, and increased numbers of neutrophils (7-9). Most patients with severe COVID-19 exhibit enhanced levels of pro-inflammatory cytokines including IL- 6 and IL-1 $\beta$, as well as MCP-1, IP-10 and G-CSF in the plasma (10). It has been 
proposed that high levels of pro-inflammatory cytokines may lead to shock, as well as respiratory failure or multiple organ failure, and several trials to assess inflammatory mediators are underway (11). However, little is known about the immunological mechanisms underlying COVID-19 severity, and the extent to which they differ from the immune response to other respiratory viruses. Furthermore, the question of whether individuals in different parts of the world respond differently to SARS-CoV-2 remains unknown. In the current study, we used a systems biological approach (mass cytometry and single cell transcriptomics of leukocytes, transcriptomics of bulk PBMCs, and multiplex analysis of cytokines in plasma), to analyze immune response in 76 COVID-19 patients and 69 age- and sex-matched controls, from two geographically distant cohorts.

\section{Results \\ Analysis of peripheral blood leukocytes from COVID-19 patients by mass cytometry}

COVID-19 infected patient samples and age- and sexmatched healthy controls were obtained from two independent cohorts, 1) from the Princess Margaret Hospital at Hong Kong University, and from 2) the Hope Clinic at Emory University in Atlanta, USA. Patient characteristics and the different assays performed are shown in Table 1. We used mass cytometry to assess immune responses to SARS-CoV-2 infection in 52 COVID-19 patients, that were confirmed positive for viral RNA by PCR, and 62 age and gender-matched healthy controls distributed between the two cohorts. To characterize immune cell phenotypes in PBMCs, we used a phospho-CyTOF panel that includes 22 cell surface markers and 12 intracellular markers against an assortment of kinases and phospho-specific epitopes of signaling molecules and H3K27ac, a marker of histone modification that drives epigenetic remodeling $(12,13)$ (table S1). The experimental strategy is described in Fig. 1A. The phospho-CyTOF identified 12 major subtypes of innate and adaptive immune cells in both cohorts, as represented in the tSNE plots (Fig. 1B). There was a striking increase in the frequency of plasmablast and effector CD8 $\mathrm{T}$ cells in all infected individuals (Fig. 1B) in both cohorts, as described recently $(6,8,14)$. Of note, the kinetics of the CD8 effector $\mathrm{T}$ cell response was prolonged and continued to increase up to day 40 post onset of the symptoms (fig. S1).

We further used manual gating to identify 25 immune cell subsets (fig. S2) and determined if there were changes in the frequency or signaling molecules of innate immune cell populations consistent between the two cohorts. There were several differences, but interestingly the frequency of plasmacytoid DCs (pDC) was significantly reduced in PBMCs of SARS-CoV-2-infected individuals in both cohorts (Fig. 1C). The kinetics of pDC response did not show an association with the time since symptom onset (fig. S1C). Neither did the observed changes correlate with clinical severity of infection (fig. S1). In addition, there was reduced expression of pS6 (phosphorylated ribosomal protein S6, a canonical target of mTOR activation (15)) in plasmacytoid DCs (pDCs) and decreased $\mathrm{IKB} \alpha$, an inhibitor of the NF-k $\beta$ signaling, in myeloid DCs (mDCs) (Fig. 1, D and E). mTOR signaling is known to mediate production of IFN- $\alpha$ in pDCs (16) suggesting that in COVID-19 patients pDCs may be impaired in their capacity to produce IFN- $\alpha$. Finally, we employed a linear modeling approach to detect features that distinguish healthy from infected individuals, and individuals based on clinical severity of COVID-19. This analysis was performed with cohort (Hong Kong or Atlanta) as a covariate to identify only features that were consistent across cohorts. The distinguishing features between healthy and infected individuals are shown in Fig. $1 \mathrm{~F}$ which includes frequencies of plasmablast and effector $\mathrm{T}$ cells, and the changes in innate immune cells described above in addition to STAT1 and other signaling events in T cells and NK cells. Of note, no features were significantly different between clinical severity groups.

We further examined the effect of various therapeutic interventions on the immune responses using samples from the Hong Kong cohort, in which some patients were treated with IFN- $\beta 1$, corticosteroids or antivirals. The infected individuals, irrespective of the intervention, showed an increased plasmablast and effector CD8 T cell frequency in comparison to healthy controls (fig. S3). However, there was an increased frequency of effector CD8 T cells (fig. S3, bottom panel, right column) and decreased pS6 signal in pDCs in antiviral treated individuals (fig. S4).

\section{COVID-19 results in functional impairment of blood myeloid cells and plasmacytoid DCs}

Given the earlier findings that mTOR signaling in pDCs mediates production of IFN- $\alpha$ in response to TLR stimulation (16) the reduced expression of pS6 in pDCs suggested that such cells may be impaired in their capacity to produce IFN$\alpha$. To test this, we performed ex vivo stimulation of PBMCs from healthy or COVID-19 infected individuals, using a mixture of synthetic Toll-like receptor (TLR) $7 / 8$ and 3 ligands, known to be expressed by viruses, and performed an intracellular staining assay to detect cytokine responses. The TLR ligands included TLR-3 and -7/8 ligands, polyIC and R848. Consistent with our hypothesis, there was reduced production of IFN- $\alpha$ in response to the TLR-stimuli in pDCs of infected individuals in comparison to healthy controls (Fig. 2A). The TNF- $\alpha$ response was also significantly reduced in pDCs of infected individuals, demonstrating that the pDCs are functionally impaired in COVID-19 infection. We also determined the ability of myeloid DCs (mDCs) and CD14 ${ }^{+}$monocytes to respond to TLR stimuli. Surprisingly, the response in 
mDCs as well as monocytes was also significantly lower in response to stimulation with a bacterial ligand cocktail (comprised of TLR-2, 4 and 5 ligands), or with the viral TLR cocktail (Fig. 2B and fig. S5). Furthermore, the reduced IKB $\alpha$ levels did not translate into enhanced NF-k $\beta$ p 65 phosphorylation as measured by p65 (Ser 529) in the same cells (Fig. $2 \mathrm{C}$ ). These results suggest that the innate immune cells in the periphery of COVID-19 infected individuals are suppressed in their response to TLR stimulation, irrespective of the clinical severity.

\section{Enhanced concentrations of cytokines and inflamma- tory mediators in plasma from COVID-19 patients}

The impaired cytokine response of myeloid cells and pDCs in response to TLR stimulation was surprising and seemingly at odds with the literature describing an enhanced inflammatory response in COVID-19 infected individuals. Several studies have described higher plasma levels of cytokines including but not limited to IL-6, TNF- $\alpha$, and CXCL10 (10, 17-19). Therefore, we evaluated cytokines and chemokines in plasma samples from the Emory cohort using the Olink multiplex inflammation panel that measures 92 different cytokines and chemokines. Of the 92 analytes measured, 71 proteins were detected within the dynamic range of the assay. Of the 71 proteins, 43 cytokines including IL-6, MCP-3 and CXCL10 were significantly up-regulated in COVID-19 infection (Fig. 3, top row, and fig. S6). These results demonstrate that plasma levels of inflammatory molecules were significantly up-regulated, despite the impaired cytokine response in blood myeloid cells and pDCs, arguing for a tissue origin of the plasma cytokines.

In addition to IL- 6 and other cytokines described previously (10), we identified three proteins that were significantly enhanced in COVID-19 infection and strongly correlated with clinical severity (Fig. 3, bottom row). They were TNFSF14 (LIGHT, a ligand of Lymphotoxin B receptor, highly expressed in human lung fibroblasts and implicated in lung tissue fibrosis and remodeling and inflammation (20)), ENRAGE (S100A12, a biomarker of pulmonary injury, and implicated in pathogenesis of sepsis-induced acute respiratory distress syndrome (ARDS) (21)) and oncostatin-M (OSM, a regulator of IL-6). Of note, the TNFSF14 is uniquely enhanced in the plasma of COVID-19 infected individuals, but not other related pulmonary infections such as influenza (flu) virus and respiratory syncytial virus (RSV) (Fig. 3). Given the striking and unappreciated observations of the enhanced plasma concentrations of TNFSF14, EN-RAGE, and OSM and their correlation to disease severity, we used an ELISA assay to independently validate these results. Consistent with the multiplex Olink analysis, we found a significant increase of these inflammatory mediators in the plasma of severe/ICU COVID-19 patients. Furthermore, we found a correlation between multiplex analysis by Olink and the ELISA results (fig. S7). These results suggest that COVID-19 infection induces a distinctive inflammatory program characterized by cytokines released from tissues, most likely the lung, but suppression of the innate immune system in the periphery. More importantly, these observations may represent novel therapeutic strategies for intervention against severe COVID-19.

\section{Single cell transcriptional response to COVID-19 infection}

To investigate the molecular and cellular processes that lead to the distinctive inflammatory program, we used cellular indexing of transcriptomes and epitopes by sequencing (CITEseq) and profiled the gene and protein expression in PBMC samples of COVD-19-infected individuals. Cryopreserved PBMC samples from a total of 12 age-matched subjects in the Emory cohort (5 Healthy controls and 7 COVID-19 patients; Table 2), were enriched for DCs, stained using a cocktail of 36 DNA-labeled antibodies (table S2), and analyzed using droplet-based single-cell gene expression profiling approaches (Fig. 4A). We performed the experiment in 2 batches and obtained transcriptomes for more than 63,000 cells, after initial preprocessing. Next, we generated a cell-bygene matrix and conducted dimensionality reduction via UMAP and graph-based clustering. Analysis of cell distribution within the UMAP between experiments revealed no major differences and we analyzed the datasets from the two experiments, together without batch correction (fig. S8). Next, we calculated the per-cell QC metrics (fig. S9), differentially expressed genes (DEGs) in each cluster compared to all other cells (fig. S10 and table S4), and the abundance of DNAlabeled antibodies in each cell (fig. S11). Using this information, we filtered low quality cells and manually annotated the clusters. After QC and cluster annotation, we retained a final dataset with 57,669 high quality transcriptomes and a median of $\sim 4,781$ cells per sample and 1,803 unique genes per cell that we used to construct the single-cell immune cell landscape of COVID-19 (Fig. 4B).

We observed several clusters that were primarily identified in COVID-19 infected individuals, including a population of plasmablasts, platelets, red blood cells and several populations of granulocytes. Interestingly, we detected clusters of T cells, and monocytes, that were characterized by the expression of interferon-response genes (ISGs) such as IFI27, IFITM3, or ISG15 (See C11-C MONO_IFN and C18-T_IFN in fig. S10). These interferon response-enriched clusters emerged uniquely in samples from COVID-19 patients (fig. S12).

To describe the unique transcriptional state of single cells from COVID-19 infected individuals, we determined the differentially expressed genes (DEGs) for cells from all COVID19-infected samples in a given cluster compared to the cells 
from all healthy individuals in the same cluster. We then analyzed these DEGs with overrepresentation analysis using blood transcriptional modules (BTMs) (22) to better understand which immune pathways are differentially regulated in patients with COVID-19 compared to healthy individuals (Fig. 4C and fig. S13). The analysis indicated a marked induction of antiviral BTMs, especially in cell types belonging to the myeloid and dendritic cell lineage. Detailed analysis of the expression pattern of the unique union of genes driving the enrichment of these antiviral pathways in monocytes and dendritic cells revealed that many ISGs were up-regulated in these cell types, (Fig. 4C, heatmap). Given our observations of muted IFN- $\alpha$ production in pDCs (Fig. 2A), we investigated the expression of genes encoding various type I and type II interferons across cell types (Fig. 4D and fig. S14). Importantly, with the exception of modest levels of IFN-Y expression in $\mathrm{T}$ and $\mathrm{NK}$ cells, we could not detect any expression of IFN- $\alpha$ and $-\beta$ genes, consistent with the functional data demonstrating impaired type I IFN production by pDCs and myeloid cells (Fig. 2). However, there was an enhanced expression of ISGs in patients with COVID-19 (Fig. 4D), despite an impaired capacity of the innate cells in the blood compartment to produce these cytokines.

Despite the lack of type 1 IFN gene expression, the presence of an ISG signature in the PBMCs of COVID-19 infected individuals, raised the possibility that low quantities of type 1 IFNs produced in the lung by SARS-CoV-2 infection (17), might circulate in the plasma and induce the expression of ISGs in PBMCs. We thus measured the concentration of IFN$\alpha$ in plasma using a highly sensitive ELISA enabled by single molecule array (SIMoA) technology. Indeed, we observed a significant increase in the concentration of IFN- $\alpha$, which peaked around day 8 post onset of symptoms and regressed to baseline levels by day 20 (Fig. 4E). Strikingly, we observed a strong correlation between the average expression levels of the ISG signature in PBMCs identified by CITE-seq analysis, and the IFN- $\alpha$ concentration in plasma (Fig. 4F). In addition, we noticed a strong temporal dependence of IFN- $\alpha$ response.

In order to investigate this further, and to independently validate the observations in the CITE-seq analysis, we performed bulk RNAseq analysis of PBMCs in an extended group of subjects (17 COVID-19 and 17 healthy samples) from the same cohort. We first evaluated if the ISG signature containing 33 genes identified in the CITE-seq data was also observed in the bulk RNAseq dataset. We observed a strong induction of the ISGs in COVID-19 subjects in comparison to healthy donors in this dataset as well (Fig. 4G). Of note, we did not detect expression of genes encoding IFN- $\alpha / \beta$, consistent with the CITE-seq and flow cytometry experiments (Fig. 2 and Fig. 4D). We also performed an unbiased analysis of an extended set of genes in the interferon transcriptional network (23) and found that these were induced in COVID-
19 subjects relative to healthy controls, as observed for the limited ISG signature (fig. S15A). Similar to the observation with CITE-seq data (Fig. 4F), there was a strong correlation between circulating IFN- $\alpha$ and the ISG response measured by the bulk transcriptomics (fig. S15B). Additionally, we analyzed the individual impact of major covariates, time, disease severity, sex, and age, on the observed ISG signature. While time emerged as the major driver of ISG signature, COVID-19 clinical severity also had an impact (Fig. 4H and fig. S15C). Taken together, these data demonstrate that early during SARS-CoV-2 infection, there are low levels of circulating IFN$\alpha$ that induces interferon-response genes in the periphery while the innate immune cells in the periphery are impaired in their capacity to produce inflammatory cytokines.

In addition to an enhanced ISG signature, the CITE-seq analysis revealed a significant decrease in the expression of genes involved in the antigen-presentation pathways in myeloid cells (Fig. 4C and fig. S13). Consistent with this, we observed a reduction in the expression of the proteins CD86 and HLA-DR on monocytes and mDCs of COVID-19 patients which was most pronounced in subjects with severe COVID19 infection (Fig. 5A and fig. S16A). HLA-DR is an important mediator of antigen-presentation and crucial for the induction of $\mathrm{T}$ helper cell responses. Using the phospho-CyTOF data from both the Atlanta and the Hong Kong cohorts, we confirmed the reduced expression of HLA-DR on monocytes and $\mathrm{mDCs}$ in patients with severe COVID-19 disease (Fig. 5B). In contrast, S100A12, the gene encoding EN-RAGE was strikingly increased in PBMCs of COVID-19 patients, while the expression of genes encoding other proinflammatory cytokines was either absent or unchanged compared to healthy controls (Fig. 5C and fig. S16B). Notably, the S100A12 expression was highly restricted to monocyte clusters (Fig. 5D) and showed a significant correlation with EN-RAGE protein levels in plasma measured by Olink (Fig. 5E). Finally, we examined if there is an association between HLA-DR and S100A12 expression in our dataset and found a strong inverse correlation between S100A12 gene expression and the genes encoding the antigen presentation machinery (HLA-DPA1, HLA-DPB1, HLA-DR, CD74) (Fig. 5F and fig. S17). Of note, the receptor for S100A12, AGER (RAGE), was expressed sparsely in PBMCs (fig. S18) suggesting that the target of EN-RAGE action was likely to be elsewhere, perhaps the lung where RAGE is known to be expressed in type 1 alveolar epithelial cells and mediate inflammation (24).

Taken together, CITE-seq analysis of PBMCs in COVD-19 patients revealed the following novel mechanistic insights: (i) Lack of expression of genes encoding type-I IFN and pro-inflammatory cytokines in PBMCs, consistent with the mass cytometry (Fig. 1C) and functional data (Fig. 2); (ii) an early but transient wave of ISG expression, which was entirely consistent with analysis of RNA-seq from bulk PBMCs (Fig. 4G 
and fig. S15A), and strongly correlated with an early burst of plasma IFN- $\alpha$ (Fig. 4F), likely of lung origin (17); (iii) and impaired expression of HLA-DR and CD86 but enhanced expression of S100A12 in myeloid cells, consistent with the mass cytometry (Fig. 5B) and Olink (Fig. 3) and ELISA (fig. S7) data, and a phenotype reminiscent of myeloid derived suppressor cells described previously (25).

\section{Severe COVID-19 infection is associated with systemic release of bacterial products}

The increased levels of proinflammatory mediators in the plasma including IL-6, TNF, TNFSF14, EN-RAGE and OSM (Fig. 3), coupled with suppressed innate immune responses in blood monocytes and DCs (Fig. 2 and fig. S5) suggested a sepsis-like clinical condition $(26,27)$. In this context, it has been previously suggested that proinflammatory cytokines and bacterial products in the plasma may play pathogenic roles in sepsis, and the combination of these factors could be important in determining patient survival $(28,29)$. Therefore, to determine whether a similar mechanism could be at play in severe COVID-19 patients, we measured bacterial DNA and lipopolysaccharide (LPS) in the plasma. Strikingly, the plasma of severe and ICU patients had significantly higher levels of bacterial DNA, as measured by PCR quantitation of bacterial 16S rRNA gene product, and LPS, as measured by a TLR4-based reporter assay (Fig. 6, A and B). Furthermore, there was a significant correlation between bacterial DNA or LPS, and the plasma levels of the inflammatory mediators IL6, TNF, MCP-3, EN-RAGE, TNFSF14 and OSM (Fig. 6C and fig. S19). These results suggest that the enhanced cytokine release may in part have been caused by increased bacterial products in the lung or in other tissues.

\section{Discussion}

We used a systems biology approach to determine host immune responses to COVID-19. Mass cytometry analysis of peripheral blood leukocytes from two independent cohorts, from Hong Kong and Atlanta revealed several common features of immune responses induced upon SARS-CoV-2 infection. There was a striking and protracted increase in the frequencies of plasmablasts and effector CD8 T cells in the peripheral blood consistent with recent studies $(6,8,14)$. Of note, the effector $\mathrm{T}$ cells continued to increase up to day 40 post symptom onset. Studies have shown that SARS-CoV-2 infection induces exhaustion and apoptosis in T cells $(30,31)$. Whether the continuing effector CD8 T cell response reflects continuous exposure to antigen, and if the cells are exhausted needs further investigation.

In contrast to robust activation of $\mathrm{B}$ and $\mathrm{T}$ cells, we observed a significant decrease in the frequency of pDCs. Furthermore, mTOR signaling in pDCs was reduced significantly in COVID-19 infected individuals, as measured by decreased
pS6 signaling by mass cytometry. These results suggest that pDCs, the major producers of type I IFNs are impaired in COVID-19 infection, consistent with studies in SARS-CoV infection (32). To determine whether the reduced mTOR signaling in pDCs resulted in impairment of type I IFN production, we stimulated cells in vitro with TLR ligands. Our results demonstrate that pDCs from COVID-19 infected patients are functionally impaired in their capacity to produce IFN- $\alpha$ in response to TLR stimulation. Taken together, the data suggest that COVID-19 causes an impaired type I IFN responses in the periphery. Administration of type I IFN has been proposed as a strategy for COVID-19 intervention (33); however, it must be noted that type I IFN signaling has been shown to elevate ACE2 expression (34) in lung cells, potentially leading to enhanced infection.

In addition to the impaired IFN- $\alpha$ production by pDCs, there was a marked diminution of the pro-inflammatory cytokines IL- 6 , TNF- $\alpha$ and IL-1 $\beta$ produced by monocytes and myeloid DCs, upon TLR stimulation (Fig. 2B). This was consistent with lack of or diminished expression of the genes encoding IL-6 and TNF in the CITE-seq analysis (Fig. 5C). These results suggest an impaired innate response in blood leukocytes of patients with COVID-19. This concept was further supported by the CyTOF and flow cytometry data showing decreased HLA-DR and CD86 expression, respectively, in myeloid cells (Fig. 5, D and E, and fig. S16). In order to obtain deeper insight into the mechanisms of host immunity to SARS-CoV-2, we performed CITE-seq single cell RNA sequencing and bulk RNA sequencing analysis in COVID-19 patients at various stages of clinical severity. Our data demonstrate that SARS-CoV-2 infection results in an early wave of IFN- $\alpha$ in the circulation that induces an ISG signature. While the ISG signature shows a strong temporal dependence in our datasets, we also find that the ISG signature is strongly induced in patients with moderate COVID-19 infection (Fig. 4G). Consistent with this, Hadjadj, et al. (5), report an enhanced expression of ISGs in moderate patients in comparison to patients with severe or critical disease. Taken together, these data suggest that SARS-CoV-2 infection induces an early, transient type I IFN production in the lung that induces ISGs in the peripheral blood, primarily in mild or moderate patients. Additionally, we observed reduced expression of genes encoding proinflammatory cytokines, as well as HLA-DR expression in myeloid cells, which was consistent with the CyTOF and flow cytometry data showing reduced HLA-DR and CD86 expression, respectively, in myeloid cells.

Our multiplex analysis of plasma cytokines revealed enhanced levels of several proinflammatory cytokines as observed previously (35), and a strong association of the inflammatory mediators EN-RAGE, TNFSF14 and OSM with the clinical severity of the disease. Importantly, the 
expression of genes encoding both TNFSF14 and OSM were down-regulated in PBMCs from COVID-19 patients with severe disease in the analysis of CITE-seq data (Fig. 5C), suggesting a tissue origin for these cytokines. The gene encoding EN-RAGE, was however, expressed at high levels in blood myeloid cells in patients with severe COVID-19 (Fig. 5, C to F), although it is also possible that EN-RAGE is also expressed in the lung. Of note, these three cytokines have been associated with lung inflammatory diseases. In particular, EN-RAGE has been shown to be expressed by CD14 ${ }^{+}$HLA-DR ${ }^{\text {lo }}$ cells, the myeloid-derived suppressor cells, and is a marker of inflammation in severe sepsis $(21,25,36)$ and its receptor, RAGE is highly expressed in type 1 alveolar cells in the lung (24). Strikingly, we observed that the classical monocytes and myeloid cells from severe COVID-19 patients in the single-cell RNAseq data expressed high levels of S100A12, the gene encoding ENRAGE, but not the typical inflammatory molecules IL- 6 and TNF- $\alpha$. These data suggest that the pro-inflammatory cytokines observed in plasma likely originate from the cells in lung tissue rather than from peripheral blood cells. Taken together with the mass cytometry data, the plasma cytokine data may be utilized to construct an immunological profile that discriminates between severe versus moderate COVID19 disease (fig. S20).

In summary, these results suggest that SARS-CoV-2 infection results in a spatial dichotomy in the innate immune response, characterized by suppression of peripheral innate immunity, in the face of proinflammatory responses reported in the lung (37). Furthermore, there is a temporal shift in the cytokine response from an early but transient type 1 IFN response to a proinflammatory response during the later and more severe stages, similar to that observed with other diseases such as influenza (38). Strikingly, there were enhanced levels of bacterial DNA and LPS in the plasma, which were positively correlated with the plasma levels of EN-RAGE, TNFSF14, OSM as well as IL-6, suggesting a role for bacterial products, perhaps of lung origin, in augmenting the production of inflammatory cytokines in severe COVID-19. The biological consequence of the impaired innate response in peripheral blood is unknown but may reflect a homeostatic mechanism to prevent rampant systemic hyperactivation, in the face of tissue inflammation. Finally, these results highlight molecules such as EN-RAGE or TNFSF14, and their receptors, which could represent attractive therapeutic target against COVID-19.

\section{REFERENCES AND NOTES}

1. Z. Wu, J. M. McGoogan, Characteristics of and Important Lessons From the Coronavirus Disease 2019 (COVID-19) Outbreak in China: Summary of a Report of 72314 Cases From the Chinese Center for Disease Control and Prevention. JAMA 323, 1239-1242 (2020). doi:10.1001/jama.2020.2648 Medline

2. CDC COVID-19 Response Team, Preliminary Estimates of the Prevalence of Selected Underlying Health Conditions Among Patients with Coronavirus Disease
2019 - United States, February 12-March 28, 2020. MMWR Morb. Mortal. Wkly. Rep. 69, 382-386 (2020). doi:10.15585/mmwr.mm6913e2 Medline

3. R. Channappanavar, S. Perlman, Pathogenic human coronavirus infections: Causes and consequences of cytokine storm and immunopathology. Semin. Immunopathol. 39, 529-539 (2017). doi:10.1007/s00281-017-0629-x Medline

4. A. Grifoni, D. Weiskopf, S. I. Ramirez, J. Mateus, J. M. Dan, C. R. Moderbacher, S. A. Rawlings, A. Sutherland, L. Premkumar, R. S. Jadi, D. Marrama, A. M. de Silva, A. Frazier, A. F. Carlin, J. A. Greenbaum, B. Peters, F. Krammer, D. M. Smith, S. Crotty, A. Sette, Targets of T Cell Responses to SARS-CoV-2 Coronavirus in Humans with COVID-19 Disease and Unexposed Individuals. Cell 181, 14891501.e15 (2020). doi:10.1016/i.cell.2020.05.015 Medline

5. J. Hadjadj, N. Yatim, L. Barnabei, A. Corneau, J. Boussier, N. Smith, H. Péré, B. Charbit, V. Bondet, C. Chenevier-Gobeaux, P. Breillat, N. Carlier, R. Gauzit, C. Morbieu, F. Pène, N. Marin, N. Roche, T.-A. Szwebel, S. H. Merkling, J.-M. Treluyer, D. Veyer, L. Mouthon, C. Blanc, P.-L. Tharaux, F. Rozenberg, A. Fischer, D. Duffy, F. Rieux-Laucat, S. Kernéis, B. Terrier, Impaired type I interferon activity and inflammatory responses in severe COVID-19 patients. Science 369, 718-724 (2020). doi:10.1126/science.abc6027 Medline

6. D. Mathew, J. R. Giles, A. E. Baxter, D. A. Oldridge, A. R. Greenplate, J. E. Wu, C. Alanio, L. Kuri-Cervantes, M. B. Pampena, K. D’Andrea, S. Manne, Z. Chen, Y. J. Huang, J. P. Reilly, A. R. Weisman, C. A. G. Ittner, O. Kuthuru, J. Dougherty, K. Nzingha, N. Han, J. Kim, A. Pattekar, E. C. Goodwin, E. M. Anderson, M. E. Weirick, S. Gouma, C. P. Arevalo, M. J. Bolton, F. Chen, S. F. Lacey, H. Ramage, S. Cherry, S. E. Hensley, S. A. Apostolidis, A. C. Huang, L. A. Vella, UPenn COVID Processing Unit, M. R. Betts, N. J. Meyer, E. J. Wherry, Deep immune profiling of COVID-19 patients reveals distinct immunotypes with therapeutic implications. Science 10.1126/science.abc8511 (2020). doi:10.1126/science. abc8511 Medline

7. C. Wu, X. Chen, Y. Cai, J. Xia, X. Zhou, S. Xu, H. Huang, L. Zhang, X. Zhou, C. Du, Y. Zhang, J. Song, S. Wang, Y. Chao, Z. Yang, J. Xu, X. Zhou, D. Chen, W. Xiong, L. Xu, F. Zhou, J. Jiang, C. Bai, J. Zheng, Y. Song, Risk Factors Associated With Acute Respiratory Distress Syndrome and Death in Patients With Coronavirus Disease 2019 Pneumonia in Wuhan, China. JAMA Intern. Med. 180, 934-943 (2020). doi:10.1001/jamainternmed.2020.0994 Medline

8. L. Kuri-Cervantes, M. B. Pampena, W. Meng, A. M. Rosenfeld, C. A. G. Ittner, A. R. Weisman, R. S. Agyekum, D. Mathew, A. E. Baxter, L. A. Vella, O. Kuthuru, S. A. Apostolidis, L. Bershaw, J. Dougherty, A. R. Greenplate, A. Pattekar, J. Kim, N. Han, S. Gouma, M. E. Weirick, C. P. Arevalo, M. J. Bolton, E. C. Goodwin, E. M. Anderson, S. E. Hensley, T. K. Jones, N. S. Mangalmurti, E. T. Luning Prak, E. J. Wherry, N. J. Meyer, M. R. Betts, Comprehensive mapping of immune perturbations associated with severe COVID-19. Sci. Immunol. 5, eabd7114 (2020). doi:10.1126/sciimmunol.abd7114 Medline

9. L. Tan, Q. Wang, D. Zhang, J. Ding, Q. Huang, Y.-Q. Tang, Q. Wang, H. Miao, Lymphopenia predicts disease severity of COVID-19: A descriptive and predictive study. Signal Transduct. Target. Ther. 5, 33 (2020). doi:10.1038/s41392-0200148-4 Medline

10. C. Huang, Y. Wang, X. Li, L. Ren, J. Zhao, Y. Hu, L. Zhang, G. Fan, J. Xu, X. Gu, Z. Cheng, T. Yu, J. Xia, Y. Wei, W. Wu, X. Xie, W. Yin, H. Li, M. Liu, Y. Xiao, H. Gao, L. Guo, J. Xie, G. Wang, R. Jiang, Z. Gao, Q. Jin, J. Wang, B. Cao, Clinical features of patients infected with 2019 novel coronavirus in Wuhan, China. Lancet 395, 497506 (2020). doi:10.1016/S0140-6736(20)30183-5 Medline

11. E. J. Giamarellos-Bourboulis, M. G. Netea, N. Rovina, K. Akinosoglou, A. Antoniadou, N. Antonakos, G. Damoraki, T. Gkavogianni, M.-E. Adami, P. Katsaounou, M. Ntaganou, M. Kyriakopoulou, G. Dimopoulos, I. Koutsodimitropoulos, D. Velissaris, P. Koufargyris, A. Karageorgos, K. Katrini, V. Lekakis, M. Lupse, A. Kotsaki, G. Renieris, D. Theodoulou, V. Panou, E. Koukaki, N. Koulouris, C. Gogos, A. Koutsoukou, Complex Immune Dysregulation in COVID-19 Patients with Severe Respiratory Failure. Cell Host Microbe 27, 992-1000.e3 (2020). doi:10.1016/j.chom.2020.04.009 Medline

12. P. Cheung, F. Vallania, H. C. Warsinske, M. Donato, S. Schaffert, S. E. Chang, M. Dvorak, C. L. Dekker, M. M. Davis, P. J. Utz, P. Khatri, A. J. Kuo, Single-Cell Chromatin Modification Profiling Reveals Increased Epigenetic Variations with Aging. Cell 173, 1385-1397.e14 (2018). doi:10.1016/i.cell.2018.03.079 Medline

13. R. Fernandez, H. Maecker, Cytokine-stimulated Phosphoflow of PBMC Using CyTOF Mass Cytometry. Biol. Protoc. 5, e1496 (2015). doi:10.21769/BioProtoc.1496 Medline 
14. A. J. Wilk, A. Rustagi, N. Q. Zhao, J. Roque, G. J. Martínez-Colón, J. L. McKechnie, G. T. Ivison, T. Ranganath, R. Vergara, T. Hollis, L. J. Simpson, P. Grant, A. Subramanian, A. J. Rogers, C. A. Blish, A single-cell atlas of the peripheral immune response in patients with severe COVID-19. Nat. Med. 26, 1070-1076 (2020). doi:10.1038/s41591-020-0944-y Medline

15. A. Arif, J. Jia, B. Willard, X. Li, P. L. Fox, Multisite Phosphorylation of S6K1 Directs a Kinase Phospho-code that Determines Substrate Selection. Mol. Cell 73, 446457.e6 (2019). doi:10.1016/j.molcel.2018.11.017 Medline

16. W. Cao, S. Manicassamy, H. Tang, S. P. Kasturi, A. Pirani, N. Murthy, B. Pulendran, Toll-like receptor-mediated induction of type I interferon in plasmacytoid dendritic cells requires the rapamycin-sensitive PI(3)K-mTOR-p70S6K pathway. Nat. Immunol. 9, 1157-1164 (2008). doi:10.1038/ni.1645 Medline

17. D. Blanco-Melo, B. E. Nilsson-Payant, W.-C. Liu, S. Uhl, D. Hoagland, R. Møller, T. X. Jordan, K. Oishi, M. Panis, D. Sachs, T. T. Wang, R. E. Schwartz, J. K. Lim, R. A. Albrecht, B. R. tenOever, Imbalanced Host Response to SARS-CoV-2 Drives Development of COVID-19. Cell 181, 1036-1045.e9 (2020). doi:10.1016/i.cell.2020.04.026 Medline

18. P. A. Mudd, J. C. Crawford, J. S. Turner, A. Souquette, D. Reynolds, D. Bender, J. P. Bosanquet, N. J. Anand, D. A. Striker, R. S. Martin, A. C. M. Boon, S. L. House, K. E. Remy, R. S. Hotchkiss, R. M. Presti, J. A. OHalloran, W. G. Powderly, P. G. Thomas, A. H. Ellebedy, Targeted Immunosuppression Distinguishes COVID-19 from Influenza in Moderate and Severe Disease. medRxiv 2020.05.28.20115667 [Preprint]. 30 May 2020. https://doi.org/10.1101/2020.05.28.20115667.

19. C. Lucas, P. Wong, J. Klein, T. B. R. Castro, J. Silva, M. Sundaram, M. K. Ellingson, T. Mao, J. E. Oh, B. Israelow, T. Takahashi, M. Tokuyama, P. Lu, A. Venkataraman, A. Park, S. Mohanty, H. Wang, A. L. Wyllie, C. B. F. Vogels, R. Earnest, S. Lapidus, I. M. Ott, A. J. Moore, M. C. Muenker, J. B. Fournier, M. Campbell, C. D. Odio, A. Casanovas-Massana, Yale IMPACT Team, R. Herbst, A. C. Shaw, R. Medzhitov, W L. Schulz, N. D. Grubaugh, C. Dela Cruz, S. Farhadian, A. I. Ko, S. B. Omer, A. Iwasaki, Longitudinal analyses reveal immunological misfiring in severe COVID19. Nature 10.1038/s41586-020-2588-y (2020). doi:10.1038/s41586-020-2588y Medline

20. R. da Silva Antunes, A. K. Mehta, L. Madge, J. Tocker, M. Croft, TNFSF14 (LIGHT) Exhibits Inflammatory Activities in Lung Fibroblasts Complementary to IL-13 and TGF- $\beta$. Front. Immunol. 9, 576 (2018). doi:10.3389/fimmu.2018.00576 Medline

21. Z. Zhang, N. Han, Y. Shen, S100A12 promotes inflammation and cell apoptosis in sepsis-induced ARDS via activation of NLRP3 inflammasome signaling. Mol. Immunol. 122, 38-48 (2020). doi:10.1016/i.molimm.2020.03.022 Medline

22. S. Li, N. Rouphael, S. Duraisingham, S. Romero-Steiner, S. Presnell, C. Davis, D. S. Schmidt, S. E. Johnson, A. Milton, G. Rajam, S. Kasturi, G. M. Carlone, C. Quinn, D. Chaussabel, A. K. Palucka, M. J. Mulligan, R. Ahmed, D. S. Stephens, H. I. Nakaya, B. Pulendran, Molecular signatures of antibody responses derived from a systems biology study of five human vaccines. Nat. Immunol. 15, 195-204 (2014). doi:10.1038/ni.2789 Medline

23. S. Mostafavi, H. Yoshida, D. Moodley, H. LeBoité, K. Rothamel, T. Raj, C. J. Ye, N. Chevrier, S.-Y. Zhang, T. Feng, M. Lee, J.-L. Casanova, J. D. Clark, M. Hegen, J.-B. Telliez, N. Hacohen, P. L. De Jager, A. Regev, D. Mathis, C. Benoist, Immunological Genome Project Consortium, Parsing the Interferon Transcriptional Network and Its Disease Associations. Cell 164, 564-578 (2016). doi:10.1016/j.cell.2015.12.032 Medline

24. E. A. Oczypok, T. N. Perkins, T. D. Oury, All the "RAGE" in lung disease: The receptor for advanced glycation endproducts (RAGE) is a major mediator of pulmonary inflammatory responses. Paediatr. Respir. Rev. 23, 40-49 (2017). doi:10.1016/i.prrv.2017.03.012 Medline

25. F. Zhao, B. Hoechst, A. Duffy, J. Gamrekelashvili, S. Fioravanti, M. P. Manns, T. F. Greten, F. Korangy, S100A9 a new marker for monocytic human myeloid-derived suppressor cells. Immunology 136, 176-183 (2012). doi:10.1111/j.13652567.2012.03566.x Medline

26. T. van der Poll, F. L. van de Veerdonk, B. P. Scicluna, M. G. Netea, The immunopathology of sepsis and potential therapeutic targets. Nat. Rev. Immunol. 17, 407-420 (2017). doi:10.1038/nri.2017.36 Medline

27. N. Watanabe, K. Kryukov, S. Nakagawa, J. S. Takeuchi, M. Takeshita, Y. Kirimura, S. Mitsuhashi, T. Ishihara, H. Aoki, S. Inokuchi, T. Imanishi, S. Inoue, Detection of pathogenic bacteria in the blood from sepsis patients using 16S rRNA gene amplicon sequencing analysis. PLOS ONE 13, e0202049 (2018). doi:10.1371/journal. pone.0202049 Medline

28. L. C. Casey, R. A. Balk, R. C. Bone, Plasma cytokine and endotoxin levels correlate with survival in patients with the sepsis syndrome. Ann. Intern. Med. 119, 771-778 (1993). doi:10.7326/0003-4819-119-8-199310150-00001 Medline

29. S. M. Opal, P. J. Scannon, J.-L. Vincent, M. White, S. F. Carroll, J. E. Palardy, N. A. Parejo, J. P. Pribble, J. H. Lemke, Relationship between plasma levels of lipopolysaccharide (LPS) and LPS-binding protein in patients with severe sepsis and septic shock. J. Infect. Dis. 180, 1584-1589 (1999). doi:10.1086/315093 Medline

30. M. Zheng, Y. Gao, G. Wang, G. Song, S. Liu, D. Sun, Y. Xu, Z. Tian, Functional exhaustion of antiviral lymphocytes in COVID-19 patients. Cell. Mol. Immunol. 17, 533-535 (2020). doi:10.1038/s41423-020-0402-2 Medline

31. B. Diao, C. Wang, Y. Tan, X. Chen, Y. Liu, L. Ning, L. Chen, M. Li, Y. Liu, G. Wang, Z. Yuan, Z. Feng, Y. Zhang, Y. Wu, Y. Chen, Reduction and Functional Exhaustion of T Cells in Patients With Coronavirus Disease 2019 (COVID-19). Front. Immunol. 11 827 (2020). doi:10.3389/fimmu.2020.00827 Medline

32. R. Channappanavar, A. R. Fehr, R. Vijay, M. Mack, J. Zhao, D. K. Meyerholz, S. Perlman, Dysregulated Type I Interferon and Inflammatory MonocyteMacrophage Responses Cause Lethal Pneumonia in SARS-CoV-Infected Mice. Cell Host Microbe 19, 181-193 (2016). doi:10.1016/i.chom.2016.01.007 Medline

33. E. Sallard, F. X. Lescure, Y. Yazdanpanah, F. Mentre, N. Peiffer-Smadja, Type 1 interferons as a potential treatment against COVID-19. Antiviral Res. 178, 104791 (2020). doi:10.1016/i.antiviral.2020.104791 Medline

34. C. G. K. Ziegler, S. J. Allon, S. K. Nyquist, I. M. Mbano, V. N. Miao, C. N. Tzouanas, Y. Cao, A. S. Yousif, J. Bals, B. M. Hauser, J. Feldman, C. Muus, M. H. Wadsworth 2nd, S. W. Kazer, T. K. Hughes, B. Doran, G. J. Gatter, M. Vukovic, F. Taliaferro, B. E. Mead, Z. Guo, J. P. Wang, D. Gras, M. Plaisant, M. Ansari, I. Angelidis, H. Adler, J. M. S. Sucre, C. J. Taylor, B. Lin, A. Waghray, V. Mitsialis, D. F. Dwyer, K. M. Buchheit, J. A. Boyce, N. A. Barrett, T. M. Laidlaw, S. L. Carroll, L. Colonna, V. Tkachev, C. W. Peterson, A. Yu, H. B. Zheng, H. P. Gideon, C. G. Winchell, P. L. Lin, C. D. Bingle, S. B. Snapper, J. A. Kropski, F. J. Theis, H. B. Schiller, L.-E. Zaragosi, P. Barbry, A. Leslie, H.-P. Kiem, J. L. Flynn, S. M. Fortune, B. Berger, R. W. Finberg, L. S. Kean, M. Garber, A. G. Schmidt, D. Lingwood, A. K. Shalek, J. OrdovasMontanes, HCA Lung Biological Network, SARS-CoV-2 Receptor ACE2 Is an Interferon-Stimulated Gene in Human Airway Epithelial Cells and Is Detected in Specific Cell Subsets across Tissues. Cell 181, 1016-1035.e19 (2020). doi:10.1016/i.cell.2020.04.035 Medline

35. G. Chen, D. Wu, W. Guo, Y. Cao, D. Huang, H. Wang, T. Wang, X. Zhang, H. Chen, H. Yu, X. Zhang, M. Zhang, S. Wu, J. Song, T. Chen, M. Han, S. Li, X. Luo, J. Zhao, Q. Ning, Clinical and immunological features of severe and moderate coronavirus disease 2019. J. Clin. Invest. 130, 2620-2629 (2020). doi:10.1172/JCl137244 Medline

36. O. M. Pena, D. G. Hancock, N. H. Lyle, A. Linder, J. A. Russell, J. Xia, C. D. Fjell, J. H. Boyd, R. E. W. Hancock, An Endotoxin Tolerance Signature Predicts Sepsis and Organ Dysfunction at Initial Clinical Presentation. EBioMedicine 1, 64-71 (2014). doi:10.1016/i.ebiom.2014.10.003 Medline

37. M. Liao, Y. Liu, J. Yuan, Y. Wen, G. Xu, J. Zhao, L. Cheng, J. Li, X. Wang, F. Wang, L. Liu, I. Amit, S. Zhang, Z. Zhang, Single-cell landscape of bronchoalveolar immune cells in patients with COVID-19. Nat. Med. 26, 842-844 (2020). doi:10.1038/s41591-020-0901-9 Medline

38. J. Dunning, S. Blankley, L. T. Hoang, M. Cox, C. M. Graham, P. L. James, C. I. Bloom, D. Chaussabel, J. Banchereau, S. J. Brett, M. F. Moffatt, A. O'Garra, P. J. M. Openshaw; MOSAIC Investigators, Progression of whole-blood transcriptional signatures from interferon-induced to neutrophil-associated patterns in severe influenza. Nat. Immunol. 19, 625-635 (2018). doi:10.1038/s41590-018-0111-5 Medline

39. M. Nowicka, C. Krieg, H. L. Crowell, L. M. Weber, F. J. Hartmann, S. Guglietta, B. Becher, M. P. Levesque, M. D. Robinson, CyTOF workflow: Differential discovery in high-throughput high-dimensional cytometry datasets. F1000 Res. 6, 748 (2017). doi:10.12688/f1000research.11622.1 Medline

40. M. E. Ritchie, B. Phipson, D. Wu, Y. Hu, C. W. Law, W. Shi, G. K. Smyth, limma powers differential expression analyses for RNA-sequencing and microarray studies. Nucleic Acids Res. 43, e47 (2015). doi:10.1093/nar/gkv007 Medline

41. E. Assarsson, M. Lundberg, G. Holmquist, J. Björkesten, S. B. Thorsen, D. Ekman, A. Eriksson, E. Rennel Dickens, S. Ohlsson, G. Edfeldt, A.-C. Andersson, P. 
Lindstedt, J. Stenvang, M. Gullberg, S. Fredriksson, Homogenous 96-plex PEA immunoassay exhibiting high sensitivity, specificity, and excellent scalability. PLOS ONE 9, e95192 (2014). doi:10.1371/journal.pone.0095192 Medline

42. J. Li, P. R. Bushel, T.-M. Chu, R. D. Wolfinger, in Batch Effects and Noise in Microarray Experiments: Sources and Solutions, A. Scherer, Ed. (Wiley Series in Probability and Statistics, Wiley, 2009), pp. 141-154.

43. F. F. Anhê, B. A. H. Jensen, T. V. Varin, F. Servant, S. Van Blerk, D. Richard, S. Marceau, M. Surette, L. Biertho, B. Lelouvier, J. D. Schertzer, A. Tchernof, A. Marette, Type 2 diabetes influences bacterial tissue compartmentalisation in human obesity. Nat Metab 2, 233-242 (2020). doi:10.1038/s42255-020-0178-9 Medline

\section{ACKNOWLEDGMENTS}

We would like to thank all participants as well as Hope Clinic and Emory Children Center staff and faculty. We would like to particularly acknowledge K. Hellmeister, A. Kay, A. Cheng, J. Traenkner, A. M. Drobeniuc, H. Macenczak, N. McNair, Y. Saklawi, A. Mehta, M. Bower, T. Girmay, E. Butler, T. Sirajud-Deen, H. Huston, D. Kleinhenz, L. Hussaini, E. Scherer, B.Johnson, J. Kleinhenz, J. Morales, V.Karmali, Y. Xu, D. Wang. We are grateful for the support of the Emory Department of Medicine and Pediatrics and for the Georgia Research Alliance. We are thankful to HIMC for assisting with sample shipments. We thank G. Kim and M. Blanco, Stanford Functional Genomics Facility, Stanford University for assistance with single-cell RNAseq and the Yerkes NHP Genomics Core (supported in part by NIH P51 OD011132). We thank the HIMC and the Parker Institute for Cancer Immunotherapy (PICl) for maintenance and access to flow cytometer. We acknowledge the support of the clinicians who facilitated this study, including Drs J.Y.H Chan, D. P-L Lau, and Y. M. Ho, and the dedicated clinical team at Infectious Diseases Centre, Princess Margaret Hospital, Hospital Authority of Hong Kong. We used equipment purchased with $\mathrm{NIH}$ grants (S100D018220 and 1S100D021763) to generate the data. Funding: This work was supported by NIH grants HIPC U19AI090023 (to B.P.), U19AI057266 (to B.P. and principal investigator Dr. Rafi Ahmed from Emory University), UH2Al132345 (to S.L.), U24Al120134 (S.E.B.), the Sean Parker Cancer Institute, the Soffer endowment (B.P.), and the Violetta Horton endowment (B.P.), Calmette and Yersin scholarship from the Pasteur International Network Association (to H.L.), National Natural Science Foundation of China (NSFC)/Research Grants Council (RGC) Joint Research Scheme (N_HKU737/18) (to C.K.P.M. and J.S.M.P), Guangzhou Medical University Highlevel University Innovation Team Training Program (Guangzhou Medical University released [2017] No.159) (to C.K.P.M. and J.S.M.P), the US National Institutes of Health (contract no. HHSN272201400006C) (to J.S.M.P) and the Research Grants Council of the Hong Kong Special Administrative Region, China (Project no. T11-712/19-N) (to J.S.M.P). Next generation sequencing services were provided by the Yerkes NHP Genomics Core which is supported in part by NIH P51 OD 011132 and the data was acquired on a NovaSeq 6000 funded by NIH S10 OD 026799. Author contributions: Conceptualization: B.P., P.S.A., and F.W.; Investigation: P.S.A., F.W., C.K.P.M., M.P., N.S., Y.F., L.B., D. W., J.C., K.L.P., G.A., C.H., and M.P.M.; Data curation and analysis: P.S.A., F.W., M.S., T.H., N.S., Y.F., D.K., A.A.U., H.T.M., and B.P.; Patient recruitment and clinical data curation: C.K.P.M., M.P., L.B., O.T.T., G.A., W.S.L., J.M.C.C., T.S.H.C., C.Y.C.C., C.H., M.P.M., H.L., E.A., S.E., N.R., and M.Peiris.; Supervision: B.P., M. Peiris, N.R., P.K., H.T.M., S.E.B., and E.A.; Data visualization: P.S.A., F.W., M.S., and T.H.; Writing: P.S.A., F.W., M.S., T.H., and B.P. All the authors read and accepted the manuscript. Funding acquisition: B.P. Competing interests: B.P. and P.S.A. are inventors on provisional patent application \#63/026,577 submitted by The Board of Trustees of the Leland Stanford Junior University, Stanford, CA that covers the use of "Therapeutic Methods of Treating COVID-19 Infections". Data and materials availability: The CITE-seq data and bulk transcriptomics data are publicly available in GEO under accession numbers GSE155673 and GSE152418, respectively. This work is licensed under a Creative Commons Attribution 4.0 International (CC BY 4.0) license, which permits unrestricted use, distribution, and reproduction in any medium, provided the original work is properly cited. To view a copy of this license, visit https://creativecommons.org/licenses/by/4.0/. This license does not apply to figures/photos/artwork or other content included in the article that is credited to a third party; obtain authorization from the rights holder before using such material.

\section{SUPPLEMENTARY MATERIALS}

science.sciencemag.org/cgi/content/full/science.abc6261/DC1

Materials and Methods

Figs. S1 to S21

Tables S1 to S4

References (39-43)

MDAR Reproducibility Checklist

5 May 2020; resubmitted 10 July 2020

Accepted 4 August 2020

Published online 11 August 2020

10.1126/science.abc6261 
A

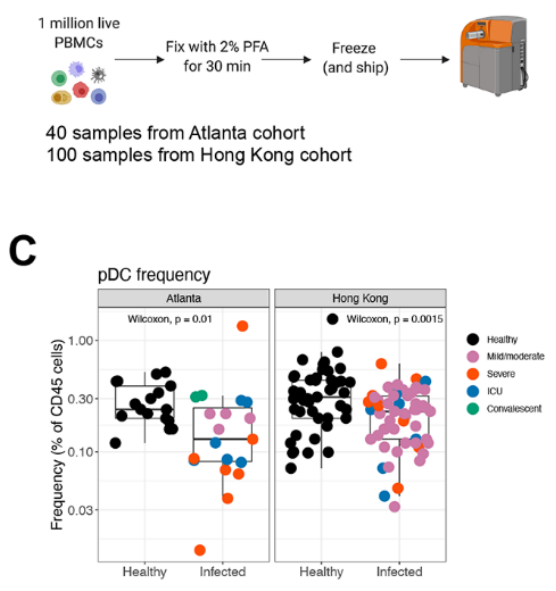

B

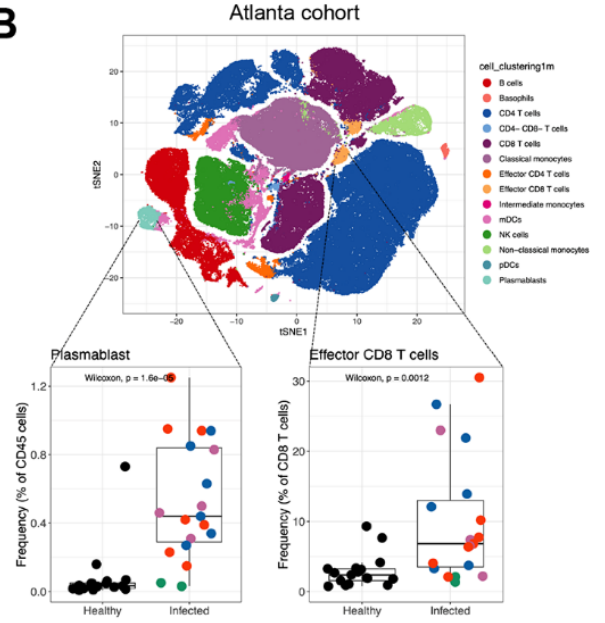

E

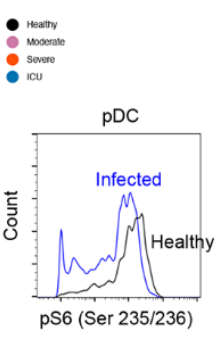

IKBa levels in mDCs
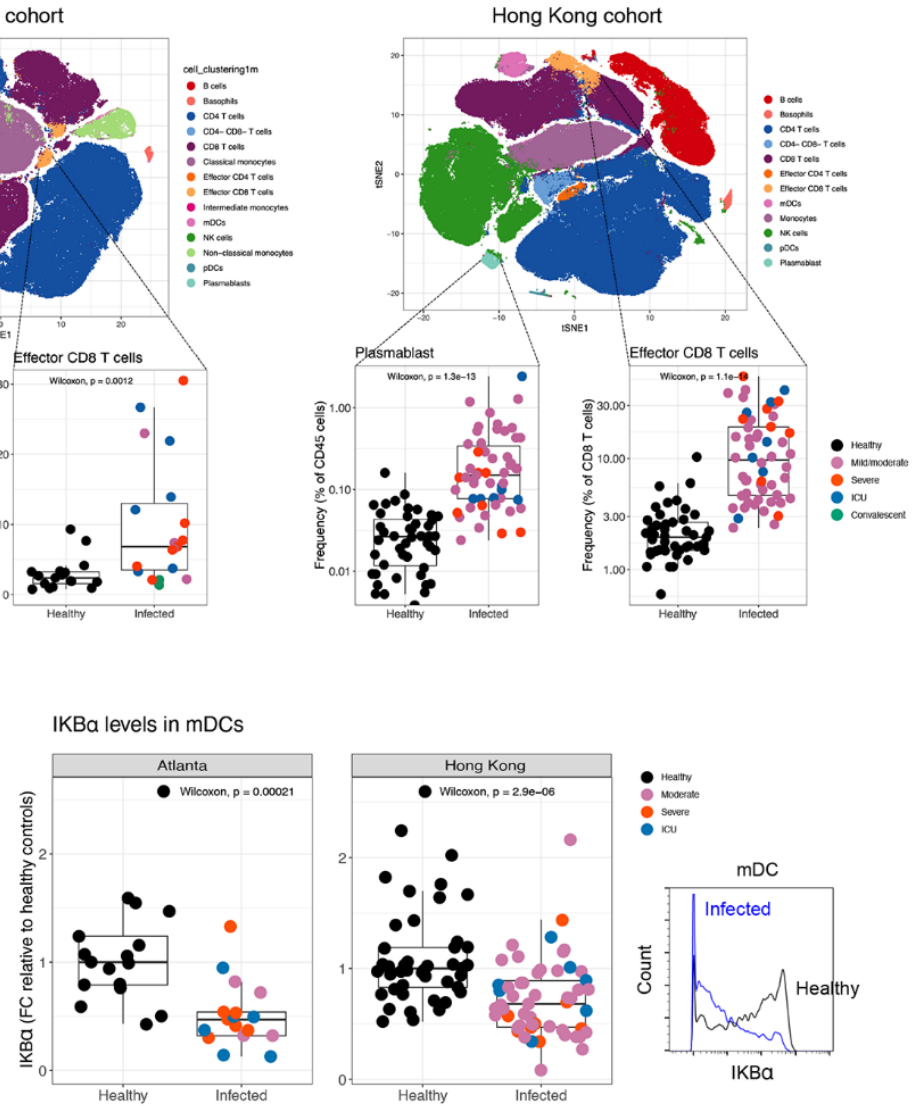

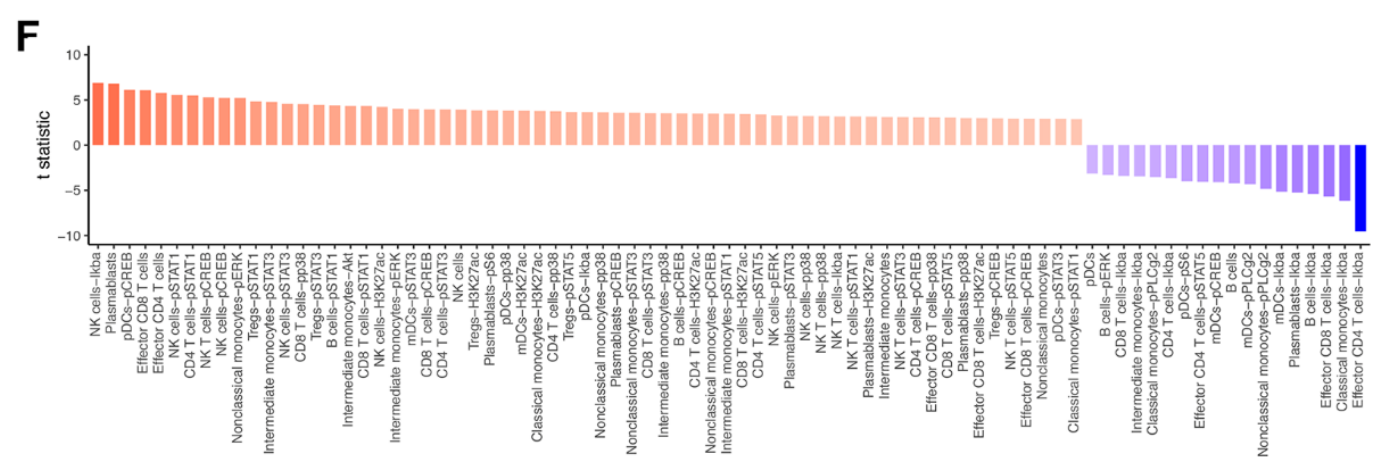

Fig. 1. Mass cytometry analysis of human peripheral blood leukocytes from COVID-19 patients. (A) A schematic representation of the experimental strategy. (B) Representation of mass cytometry identified cell clusters visualized by $\mathrm{t}$-SNE in two-dimensional space. The box plots on the bottom show frequency of plasmablasts (CD3- CD20- CD56- HLA$\mathrm{DR}^{+}$CD14- CD16- CD11c- CD123- CD19lo CD27 hi CD28 hi) and effector CD8 T cells (CD3+ CD8 ${ }^{+}$CD38 ${ }^{\text {hi }}$ HLA-DR $^{\text {hi }}$ ) in both cohorts. (C) Frequencies of pDCs (CD3- CD20 CD56- HLA-DR+ CD14- CD16- CD11c- CD123+) in healthy and infected COVID-19 individuals in both cohorts. ( $D$ and $E$ ) Box plots showing fold change of pS6 staining in pDCs (D) and IKB $\alpha$ staining in $\mathrm{mDCs}(\mathrm{E})$ relative to median of healthy controls. The histograms on the right depict representative staining of the same. (F) Distinguishing features (FDR $<0.01$ ) via linear modeling analysis of the mass cytometry data between healthy and infected subjects. In all the box plots, the boxes show median, upper and lower quartiles. The whiskers show 5 - 95 percentiles. Each dot represents an individual sample (Healthy: $n=17$ and 45; Infected: $n=19$ and 54, for Atlanta and Hong Kong cohorts, respectively). For the tSNE analysis, $n=34$ and 60 for Atlanta and Hong cohorts, respectively. Colors of the dots indicate severity of clinical disease as shown in the legends. The differences between the groups was measured by Mann-Whitney rank sum test (Wilcoxon, paired = FALSE). The p-values depicting significance are shown within the box plots. 
A Cytokine responses in pDCs
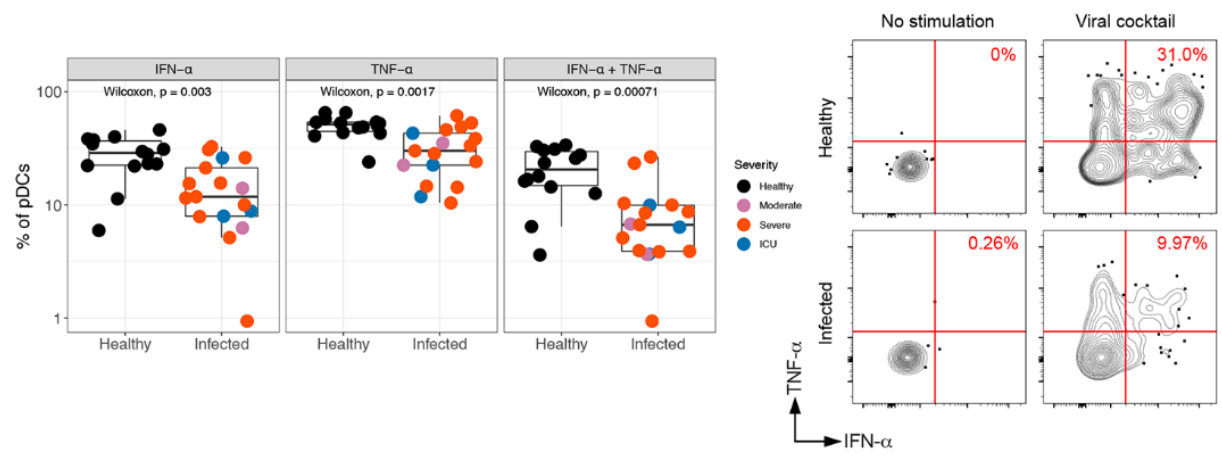

B Cytokine responses in myeloid DCs
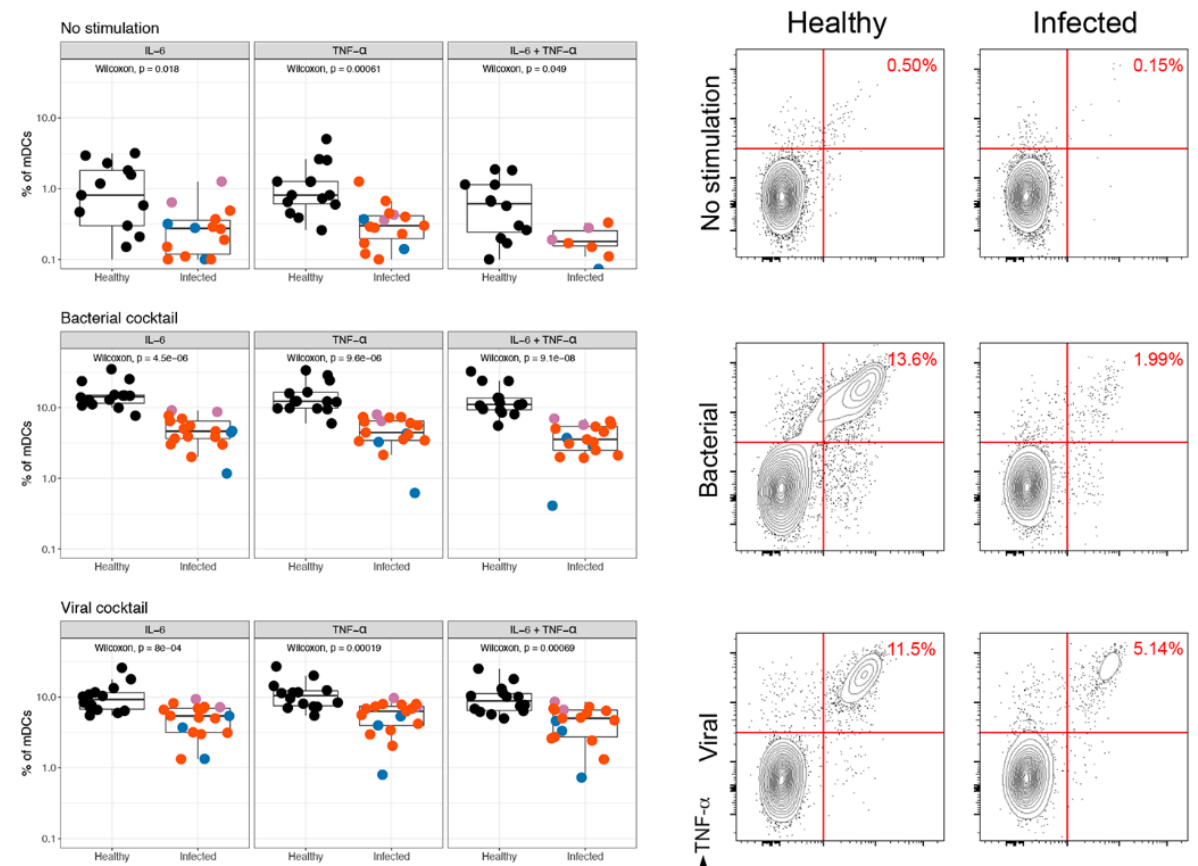

C p65(Ser529) in mDCs
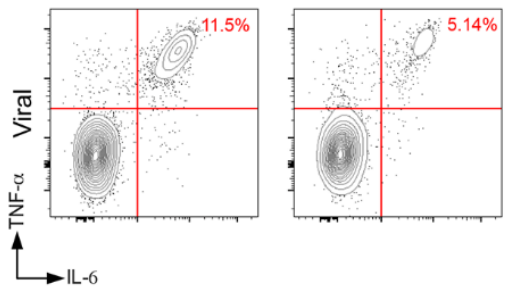

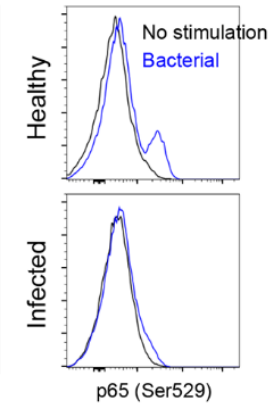

Fig. 2. Flow cytometry analysis of ex vivo stimulated human peripheral blood leukocytes from COVID-19 patients. (A) Box plots showing fraction of pDCs in PBMCs of healthy or infected donors (CD3 CD2O-

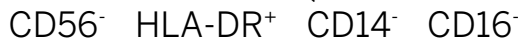
CD11c $\mathrm{CD}^{-} 23^{+}$) producing IFN$\alpha$, TNF- $\alpha$ or IFN- $\alpha+$ TNF- $\alpha$ in response to stimulation with the viral cocktail (polylC + R848). The contour plots on the right show IFN- $\alpha$, TNF- $\alpha$ or IFN$\alpha+$ TNF- $\alpha$ staining in pDCs. (B) Box plots showing fraction of $\mathrm{mDCs}$ in PBMCs of healthy or infected donors (CD3 CD20 CD56 $\mathrm{HLA}^{-\mathrm{DR}^{+}} \mathrm{CD} 14 \mathrm{CD} 16$ $\left.\mathrm{CD}^{2} 3^{+} \mathrm{CD} 11 \mathrm{C}^{-}\right)$producing IL-6, TNF- $\alpha$ or IL- $6+$ TNF- $\alpha$ in response to no stimulation (top panel), bacterial cocktail (middle panel, Pam3CSK4, LPS and Flagellin) or viral cocktail (bottom panel, polylC + R848). The flow cytometry plots on the right are representative plots gated on mDCs showing IL-6, TNF- $\alpha$ or IL-6+TNF- $\alpha$ response. (C) Fold change of NF-k $\beta$ p65 (Ser529) staining in PBMCs stimulated with bacterial cocktail relative to no stimulation in healthy and infected donors to showing reduced induction of p65 phosphorylation in infected individuals. The histograms show representative flow cytometry plots of p65 staining in myeloid DCs. In all the box plots, the boxes show median, upper and lower quartiles. The whiskers show 5 - 95 percentiles. Each dot represents an Atlanta cohort patient $(\mathrm{n}=14$ and 17 for healthy and infected, respectively). Colors of the dots indicate severity of clinical disease as shown in the legends. The differences between the groups was measured by MannWhitney rank sum test. The $\mathrm{p}$ values depicting significance are shown within the plots. 

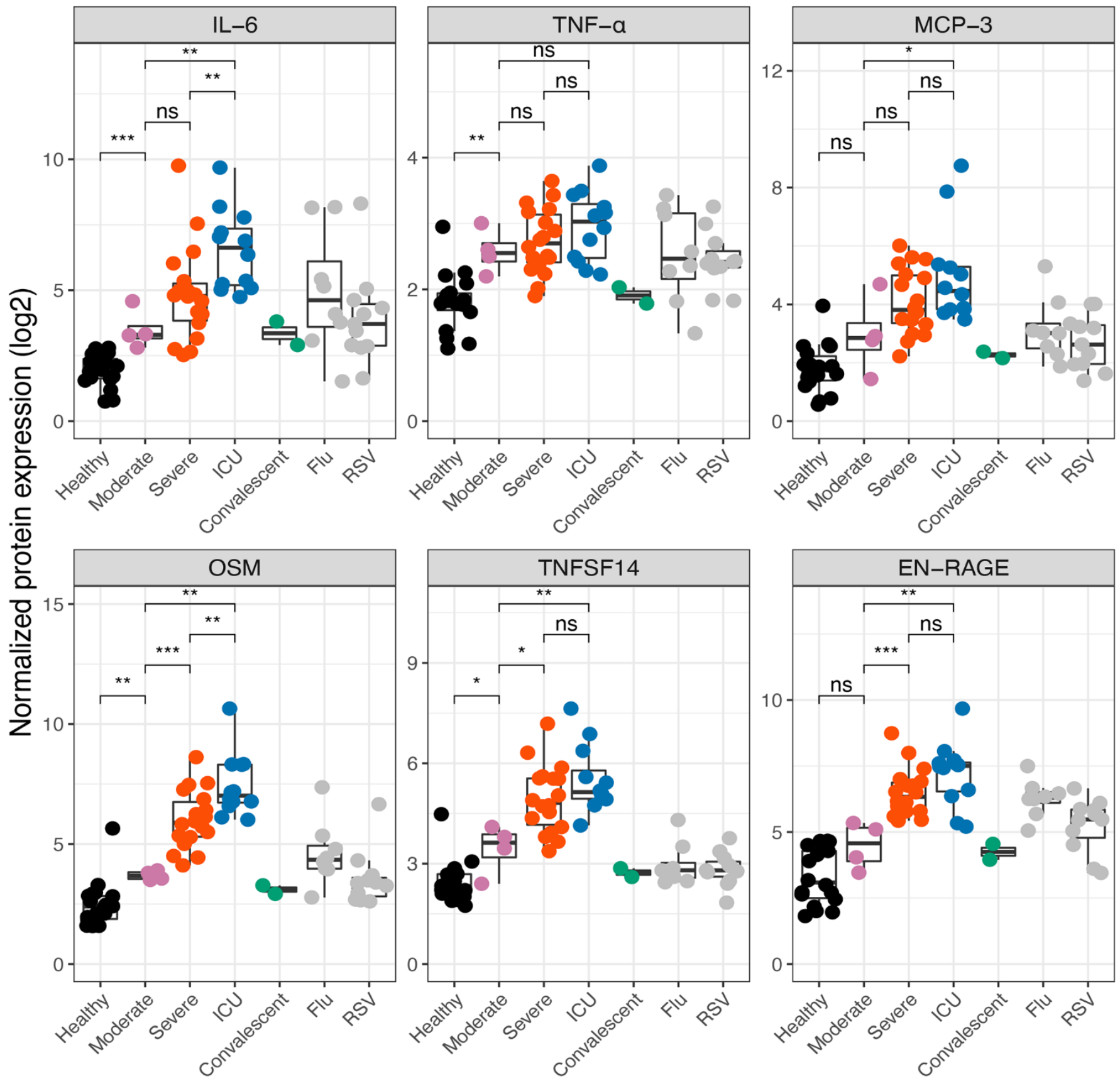

Infection severity

Fig. 3. Multiplex analysis of cytokines in the plasma of COVID-19 patients. Cytokine levels in plasma of healthy or infected individuals. The infected individuals are further classified based on severity of clinical COVID-19 disease. The normalized protein expression values plotted on the $Y$-axis is an arbitrary unit defined by Olink Proteomics to represent Olink data. In all the box plots, the boxes show median, upper and lower quartiles. The whiskers show $5-95$ percentiles. Each dot represents an Atlanta cohort sample $(n=18$ healthy, 4 moderate, 18 severe, $12 \mathrm{ICU}, 2$ convalescent, 8 Flu and $11 \mathrm{RSV}$ ). Colors of the dots indicate severity of clinical disease as shown in the legends. The differences between the groups was measured by Mann-Whitney rank sum test (Wilcoxon, paired $=$ FALSE, ${ }^{*} p<0.05 ;{ }^{* *} p<0.01,{ }^{* *} p<0.001$ ). 
A Experiment layout

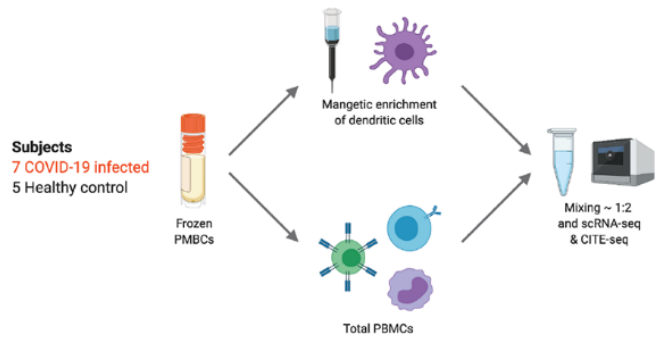

B CITE-seq of COVID-19 ( $n=7)$ and healthy $(n=5)$ subjects

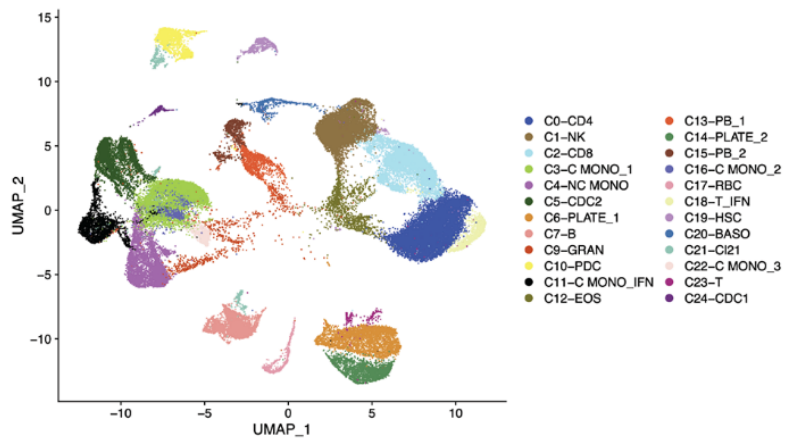

C Top BTMs enriched in DEGs COVID-19 $(n=7)$ vs healthy $(n=5)$ for each cluster

CITE-seq of COVID-19 $(n=7)$ and healthy $(n=5)$ subjects
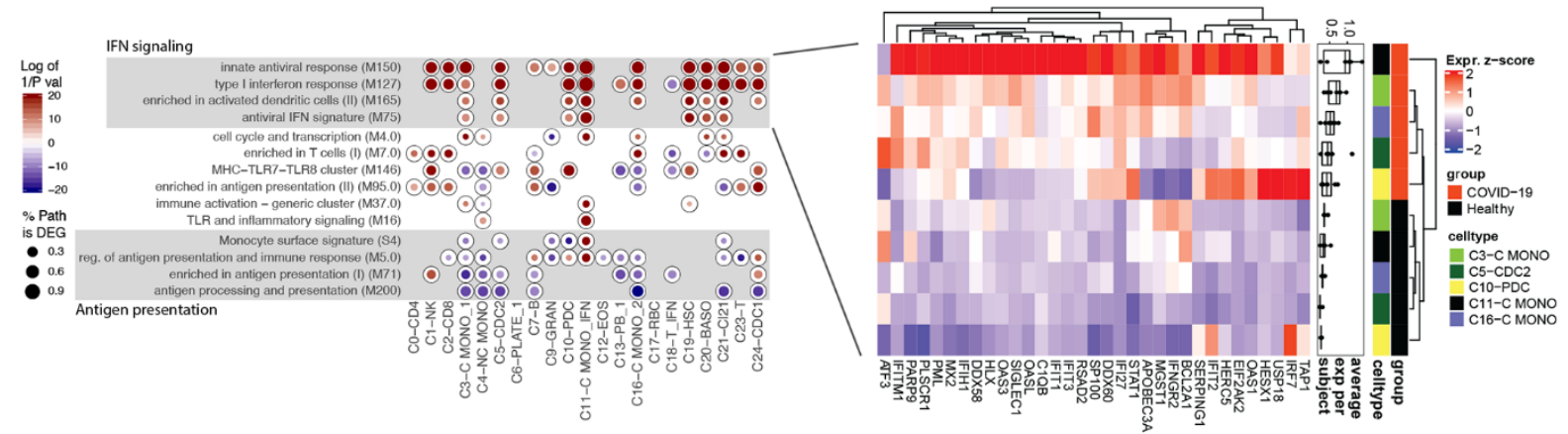

D CITE-seq of COVID-19 $(n=7)$ and healthy $(n=5)$ subjects

IFNA2

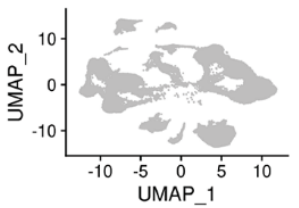

IFI27

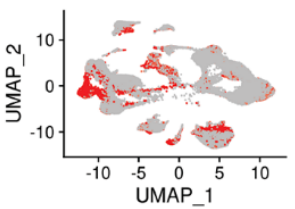

F CITE-seq vs IFNa ELISA
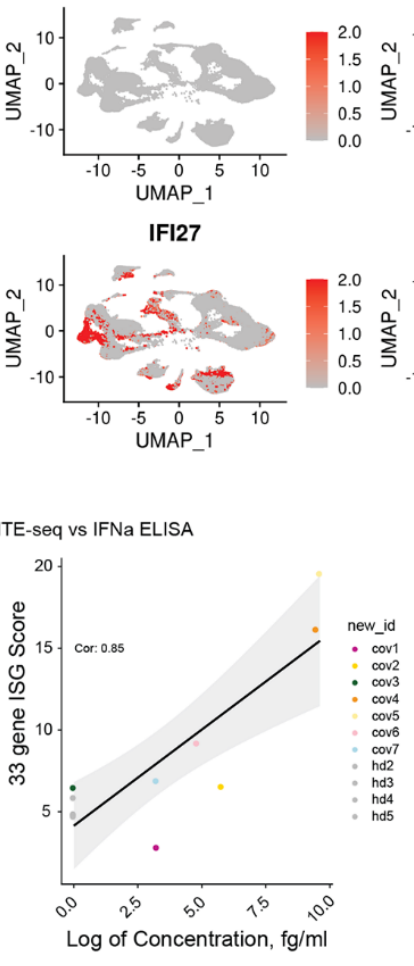

IFNB1

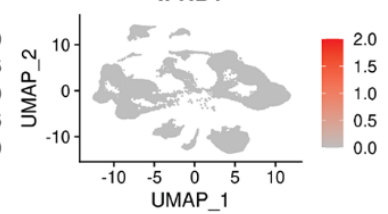

IFITM3

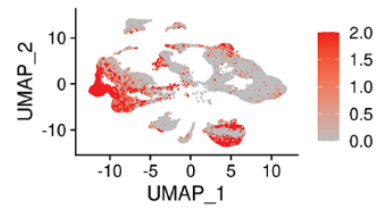

G Bulk RNA-seq of total PBMCs

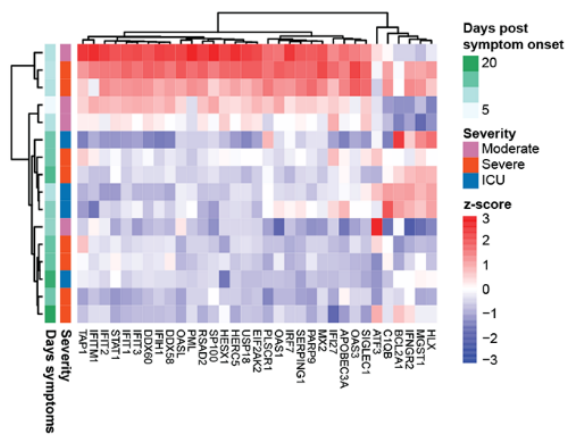

E IFNa ELISA of serum plasma

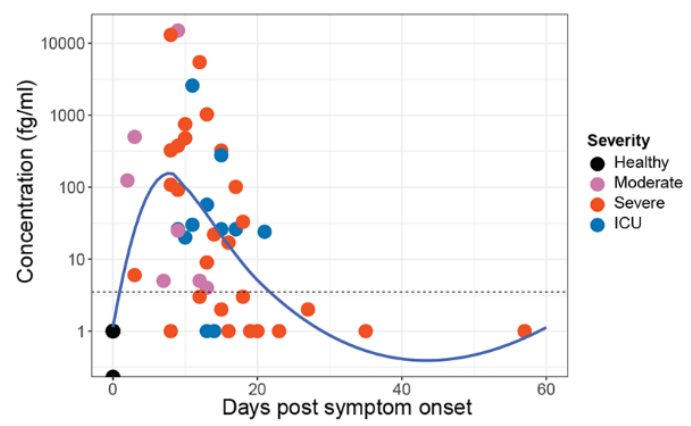

H Bulk RNA-seq of total PBMCs

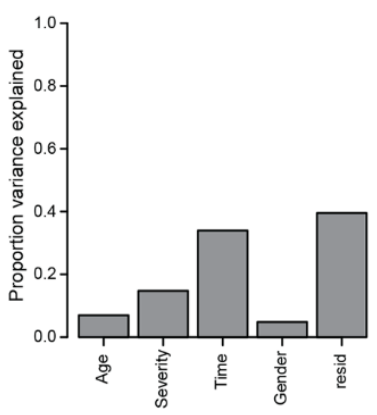


Fig. 4. Early, transient Interferon-stimulated gene (ISG) expression in COVID-19 infection. (A) A schematic representation of DC enrichment strategy used in CITE-seq analysis. (B) UMAP representation of PBMCs from all analyzed samples $(n=12)$ colored by manually annotated cell type. (C) Pairwise comparison of genes from healthy individuals $(n=5)$ and COVID-19-infected patients $(n=$ 7) was conducted for each cluster. Differentially expressed genes were analyzed for overrepresentation of BTM modules. The ringplot shows overrepresented pathways in up- and down-regulated genes of each cluster. The heatmap on the right shows average expression levels of 33 ISGs derived from the enriched BTM modules in different cell clusters of healthy $(n=5)$ and COVID-19 subjects $(n=7)$. (D) UMAP representation of PBMCs from all analyzed samples showing the expression levels of selected interferon and interferon-stimulated genes. (E) Kinetics of circulating IFN- $\alpha$ levels $(\mathrm{pg} / \mathrm{ml}$ ) in plasma measured using SIMoA technology ( $n=18$ healthy and 40 COVID-19 infected patients). (F) Correlation between circulating IFN- $\alpha$ levels in plasma and the average expression of ISGs measured by CITE-seq analysis. (G) Hierarchically clustered heatmap of expression of the CITE-seq ISG signature (C) in the bulk RNAseq dataset, performed using an extended group of subjects ( $n=17$ healthy and 17 COVID-19 infected samples). Colors represent gene-wise z-scores. $(\mathrm{H})$ Bar chart representing the proportion of variance in CITE-seq ISG signature expression explained by the covariates in X-axis via PVCA. 
A Flow: HLA-DR levels (CITE-seq samples)

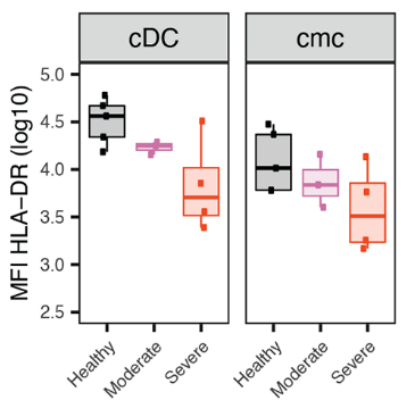

C Bulk RNA-seq of total PBMCs
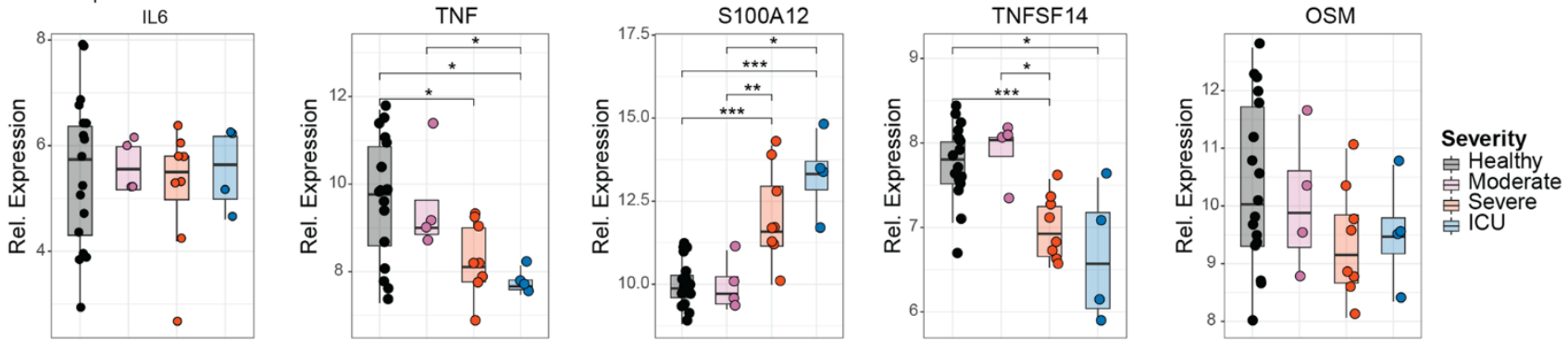

B CYTOF: MDC HLA-DR levels

CYTOF: C mono HLA-DR levels
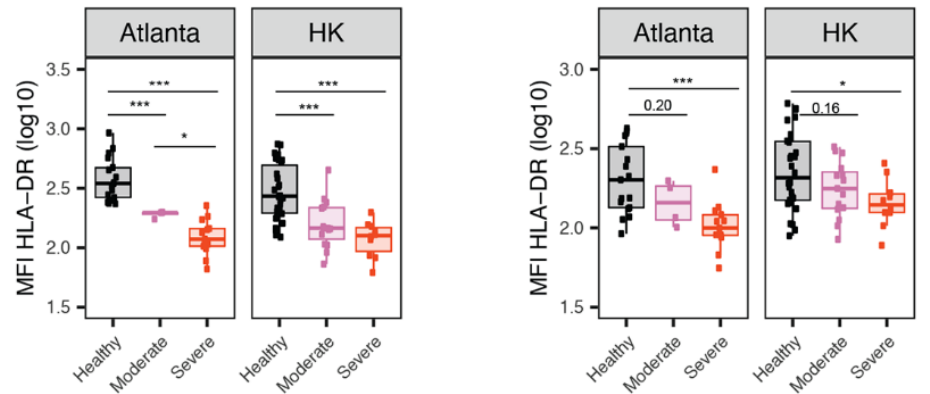

D CITE-seq

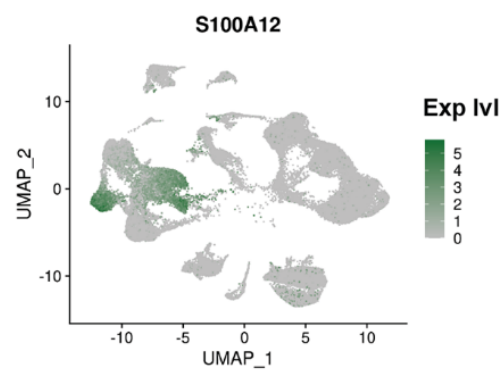

E S100A12 CITE-seq vs EN-RAGE plasma Ivls

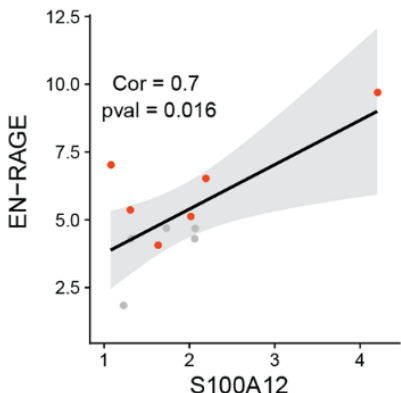

F CITE-seq, myeloid cells

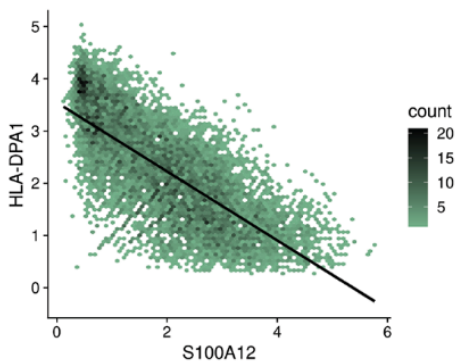

Fig. 5. Attenuated inflammatory response in peripheral innate immune cells from COVID-19 patients. (A) Flow cytometry analysis of PBMCs analyzed in parallel to the CITE-seq experiment. Shown is the $\log _{10}$ median fluorescence intensity of HLA-DR expression. (B) Median intensity of HLA-DR expression in phospho-CyTOF experiment from Fig. 1. N: HK (Healthy $=30$, Moderate $=15$, Severe $=10)$, Atlanta (Healthy $=17$, Moderate $=4$, Severe $=13$ ). The boxes indicate median, upper and lower quartiles. The whisker length equals $1.5 x$ the interquartile range. (C) Relative expression of genes encoding different cytokines in the bulk RNAseq dataset. The boxes show median, upper and lower quartiles and the whiskers show $5-95$ percentiles. (D) UMAP representation of S100A12 expression in PBMCs from all samples analyzed by CITE-seq. (E and F) Correlation analysis of S100A12 expression in cells from myeloid and dendritic cell clusters (C MONO_1, NC MONO, CDC2, PDC, C MONO_IFN, C MONO_2, C MONO_3) with EN-RAGE levels in plasma (E) or HLA-DPA1 expression in the same clusters ( $F)(n=5$ healthy and 7 COVID-19 subjects). The statistical significance between the groups in (B) and (C) was determined by two-sided Mann-Whitney rank-sum test ${ }^{*} p<0.05$; ${ }^{*} p<0.01$, ${ }^{* *} p<0.001$. 

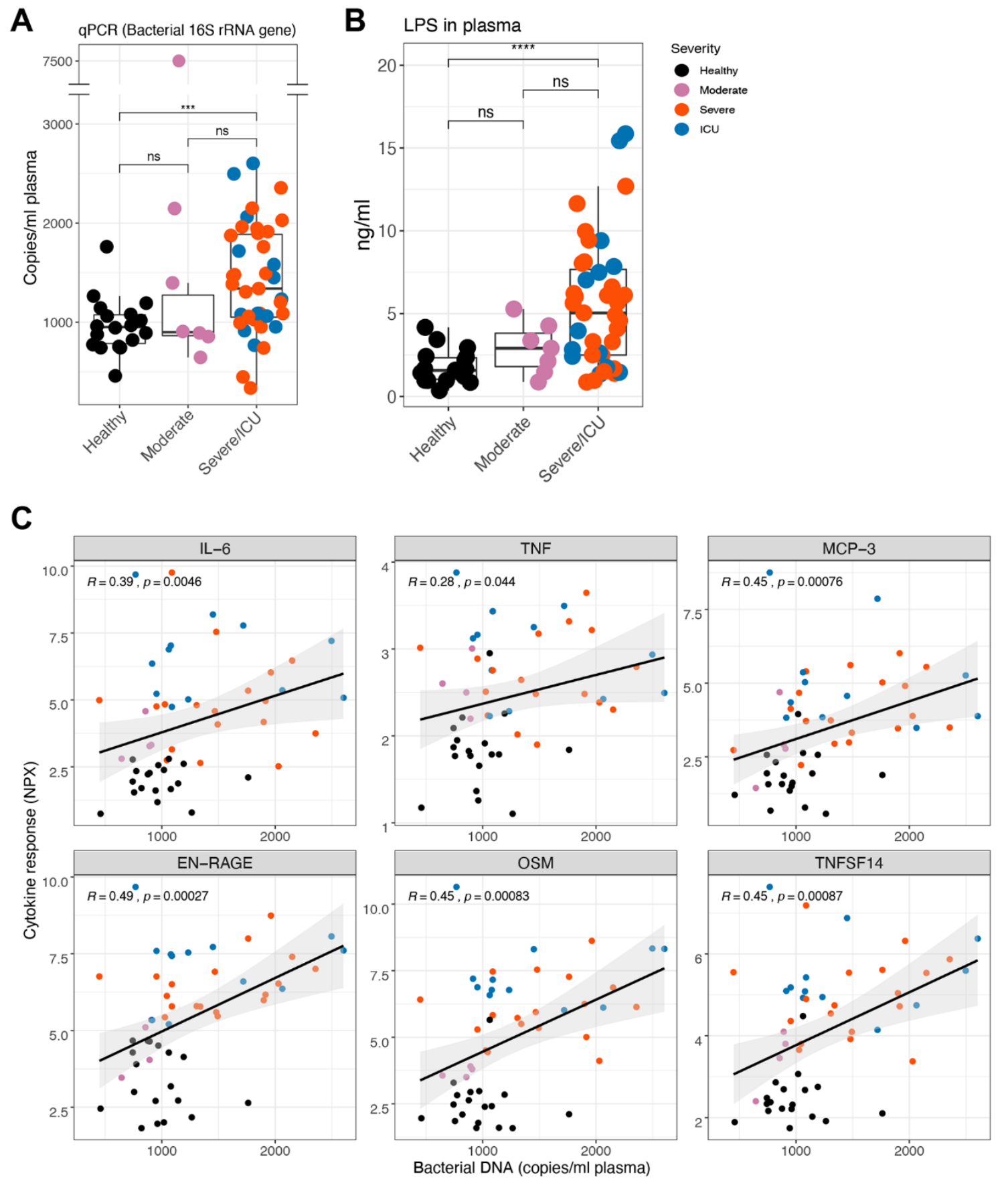

Fig. 6. Systemic release of microbial products in severe COVID-19 infection. (A to C) Box plots showing bacterial 16S rRNA gene (A) and LPS (B) measured in plasma of healthy or infected individuals (C) Spearman's correlation between cytokines and bacterial DNA measured in plasma. Each dot represents a sample ( $n=18$ and 51 for healthy and infected, respectively). Colors of the dots indicate severity of clinical disease as shown in the legends. The boxes show median, upper and lower quartiles in the box plots. The whiskers show $5-95$ percentiles. The differences between the groups was measured by Mann-Whitney rank sum test $\left({ }^{* *} p<0.001\right)$. 
Table 1. Patient characteristics and number of samples used in different assays.

\begin{tabular}{|c|c|c|}
\hline Characteristics & Hong Kong cohort & Atlanta cohort \\
\hline \multicolumn{3}{|l|}{ Number of subjects } \\
\hline COVID-19 (n) & 36 patients* & 40 patients* \\
\hline Flu/RSV (n) & NA & 16 patients \\
\hline Healthy (n) & 45 individuals & 24 individuals \\
\hline \multicolumn{3}{|l|}{ Age } \\
\hline COVID-19 (Median, range) & $55(18-80)$ & $56(25-94)$ \\
\hline Flu/RSV (n) & NA & $66(51-86)$ \\
\hline Healthy & $53(21-69)$ & $52(23-91)$ \\
\hline \multicolumn{3}{|l|}{ Gender } \\
\hline COVID-19 (Male, \%) & $58 \%$ & $55 \%$ \\
\hline Flu/RSV (Male, \%) & NA & $31 \%$ \\
\hline Healthy (Male, \%) & $58 \%$ & $42 \%$ \\
\hline \multicolumn{3}{|c|}{ Clinical severity of COVID-19 patients } \\
\hline Mild/moderate & $75 \%$ & $18 \%$ \\
\hline Severe (no ICU) & $14 \%$ & $60 \%$ \\
\hline ICU & $11 \%$ & $18 \%$ \\
\hline \multicolumn{3}{|c|}{ Clinical severity of Flu/RSV patients } \\
\hline Mild/moderate & NA & $37.5 \%$ \\
\hline Severe (no ICU) & NA & $37.5 \%$ \\
\hline ICU & NA & $31 \%$ \\
\hline \multicolumn{3}{|l|}{ Intervention } \\
\hline Interferon- $\beta 1$ & $20 \%$ & NA \\
\hline Corticosteroids & $19 \%$ & NA \\
\hline Antivirals & $61 \%$ & NA \\
\hline \multicolumn{3}{|c|}{ Assays using COVID-19 samples } \\
\hline Phospho CyTOF & $\begin{array}{l}54 \text { PBMC samples ( } 36 \text { pa- } \\
\text { tients) }\end{array}$ & $\begin{array}{l}19 \text { PBMC samples (16 pa- } \\
\text { tients) }\end{array}$ \\
\hline In vitro stimulation & NA & $\begin{array}{l}17 \text { PBMC samples (15 pa- } \\
\text { tients) }\end{array}$ \\
\hline Olink proteomics & NA & $\begin{array}{l}36 \text { plasma samples ( } 29 \text { pa- } \\
\text { tients) }\end{array}$ \\
\hline CITE-seq & NA & 7 PBMC samples (7 patients) \\
\hline Bulk RNAseq & NA & $\begin{array}{l}17 \text { PBMC samples (15 pa- } \\
\text { tients) }\end{array}$ \\
\hline Bacterial products & NA & $\begin{array}{l}51 \text { plasma samples ( } 40 \text { pa- } \\
\text { tients) }\end{array}$ \\
\hline \multicolumn{3}{|c|}{ Assays using Flu/RSV samples } \\
\hline Phospho CyTOF & NA & 4 PBMC samples (4 patients) \\
\hline Olink proteomics & NA & $\begin{array}{l}19 \text { plasma samples (16 pa- } \\
\text { tients) }\end{array}$ \\
\hline
\end{tabular}

*Some patients have blood from multiple time points. 
Table 2. Detailed characteristics of patient samples used in the CITE-seq analysis.

\begin{tabular}{lllccccc}
\hline ID & Infection & Response & ICU & Day & Age & Sex & Ethnicity \\
\hline cov1 & COVID-19 & Severe, dec. & Y & 15 & 60 & $\mathrm{~F}$ & $\mathrm{~B}$ \\
cov2 & COVID-19 & Severe & $\mathrm{N}$ & 15 & 75 & $\mathrm{~F}$ & W \\
cov3 & COVID-19 & Severe & $\mathrm{N}$ & 16 & 59 & $\mathrm{M}$ & $\mathrm{B}$ \\
cov4 & COVID-19 & Severe & $\mathrm{N}$ & 8 & 48 & $\mathrm{M}$ & $\mathrm{B}$ \\
cov5 & COVID-19 & Moderate & $\mathrm{N}$ & 9 & 53 & $\mathrm{~F}$ & $\mathrm{~B}$ \\
cov6 & COVID-19 & Moderate & $\mathrm{N}$ & 2 & 75 & $\mathrm{~F}$ & $\mathrm{~W}$ \\
cov7 & COVID-19 & Moderate & $\mathrm{N}$ & 9 & 47 & $\mathrm{~F}$ & $\mathrm{~B}$ \\
hd1 & Healthy & & & & 84 & $\mathrm{~F}$ & $\mathrm{~W}$ \\
hd2 & Healthy & & & & 68 & $\mathrm{~F}$ & W \\
hd3 & Healthy & & & & 38 & $\mathrm{M}$ & W \\
hd4 & Healthy & & & & 90 & $\mathrm{M}$ & W \\
hd5 & Healthy & & & & 70 & $\mathrm{~F}$ & W \\
\hline
\end{tabular}

